\title{
Publisher Correction: Computational optimization of electric fields for better catalysis
} design

Valerie Vaissier Welborn, Luis Ruiz Pestana and Teresa Head-Gordon (D)

Correction to: Nature Catalysis https://doi.org/10.1038/s41929-018-0109-2, published online 3 September 2018.

In the version of this Perspective originally published, in equation (1) and the sentence immediately following it, the vectors ' $\bar{\mu}$ ' and ' $\bar{E}$ ' should have appeared as ' $\boldsymbol{\mu}$ ' and 'E', in addition, in the equation, the cross product should have been the dot product; the equation should have read:

$$
\Delta G_{\text {elec }}^{\ddagger}=\left(\boldsymbol{\mu}_{\mathrm{EL}}^{\dagger} \cdot \mathbf{E}_{\mathrm{EL}}^{\dagger}-\boldsymbol{\mu}_{\mathrm{EL}} \cdot \mathbf{E}_{\mathrm{EL}}\right)
$$

Furthermore, in Fig. 1, ' $\bar{E}_{\mathrm{EL}}$ ' should have been ' $\mathrm{E}_{\mathrm{EL}^{\dagger}}^{\dagger}$ '. These errors have now been corrected.

Published online: 27 September 2018

https://doi.org/10.1038/s41929-018-0161-y 